\title{
Interproximal biofilm removal by intervallic use of a sonic toothbrush compared to an oral irrigation system
}

\author{
Pune N Tawakoli ${ }^{*}$, Bärbel Sauer ${ }^{1}$, Klaus Becker ${ }^{1}$, Wolfgang Buchalla ${ }^{2}$ and Thomas Attin ${ }^{1}$
}

\begin{abstract}
Background: The purpose of this in-vitro study was to investigate the potential of biofilm removal in interproximal tooth regions using intervallic cleaning with an oral irrigator or a sonic toothbrush.

Methods: Three-species biofilms (Streptococcus mutans (OMZ 918), Streptococcus oralis SK 248 (OMZ 60), Actinomyces naes/undii (OMZ 745)) were grown on hydroxyapatite discs for 3 days in culture media. Every 24 h, specimens were incubated for $15 \mathrm{~min}$ in resazurin solution (i.e., culture medium and $10 \% \mathrm{v} / \mathrm{v}$ alamarBlue ${ }^{\oplus}$ ) to measure the metabolic activity with a fluorescence spectrophotometer in relative fluorescence units (rfu) at baseline. Then, specimens were fixed in interproximal holding devices and underwent treatment with an oral irrigator (WF; Waterpik ${ }^{\circledast}$ Sensonic WP-100E), an active sonic toothbrush (WPa), or an inactive sonic toothbrush (WPi; Waterpik ${ }^{\circledR}$ Sensonic SR-3000E) for $10 \mathrm{~s}$ ( $n=18$ /group). Untreated biofilms served as controls (CO). After treatment, bacterial activity was re-measured, and specimens were re-grown in fresh medium for $24 \mathrm{~h}$ until next cleaning procedure. Altogether, cleaning was repeated in intervals of three treatment days $(\mathrm{d} 1, \mathrm{~d} 2, \mathrm{~d} 3)$. After $\mathrm{d} 3$, SEM images were taken $(n=8)$ and CFU was measured $(n=3)$. Metabolic activity was analyzed for each disc separately, rfu values were averaged for $\mathrm{d} 1$ to compare initial biofilm stability, and ratios of baseline and post-treatment values were compared. Results were analyzed using ANOVA with the post-hoc Scheffé test, or Kruskal-Wallis with post-hoc Mann-Whitney test.
\end{abstract}

Results: Median baseline rfu-values of d1 resulted in $7821.8 \mathrm{rfu}$ (interquartile range $=5114.5$ ). Highest reduction in metabolic activity was recorded significantly for the oral irrigator used for $10 \mathrm{~s}$ (residual activity per day d1: WF $17.9 \%$, WPa $58.8 \%$, WPi 82.5 \%, CO 89.6 \%; d2: WF $36.8 \%$, WPa 85.2 \%, WPi $82.5 \%$, CO 90.0 \%; d3: WF 17.2.\%, WPa 79.6 \%, WPi $96.3 \%$, CO $116.3 \%$ ). SEM images of untreated specimens (CO) and specimens treated with the sonic toothbrush (WPa and WPi) showed huge amounts of biofilm, while oral irrigator-treated specimens (WF) revealed barely any bacteria. CFU data confirmed the graduations between the groups.

Conclusions: Cleaning of interproximal regions achieved better success with an oral irrigator as compared to the use of a sonic toothbrush. (350/ 350 words)

Keywords: alamarBlue assay, Intervallic biofilm removal, Interproximal biofilm, Oral irrigation system, Sonic toothbrush

\section{Background}

Caries and periodontitis are caused by bacterial biofilms, accumulating on tooth surfaces and oral soft tissues. Since most oral hygiene devices do not sufficiently reach all niches and angles in the oral cavity mechanically, interproximal regions are often only affected by the

\footnotetext{
* Correspondence: nina.tawakoli@zzm.uzh.ch

${ }^{1}$ Clinic of Preventive Dentistry, Periodontology and Cariology, University of Zurich, Center of Dental Medicine, 8032 Zurich, Switzerland

Full list of author information is available at the end of the article
}

properties of toothpaste slurry and the hydrodynamic forces produced during tooth brushing. To determine the highest hydrodynamic effects, many studies investigated the effect of sonic and manual tooth brushing on biofilms as well as differences within various types of sonic toothbrushes. Depending on the sonic toothbrushes' type, side-to-side toothbrushes result more often in higher biofilm reduction than $50 \%$, while multidimensional toothbrushes remove less biofilm [1-3]. Comparing different side-to-side sonic toothbrushes 
among each other shows significant differences between the models ranging from 9 to $80 \%$ [4]. However, until now, most investigations on the so called 'non-contact biofilm removal' were performed not using interproximal devices, but e.g. sonic toothbrushes, installed with defined distances directly adjusted towards the center of the biofilm coated disc surface [3-7]. Using this approach, the hydrodynamic forces, formed by the oral devices have direct access to the biofilm surface and strike, depending on the distance, with full intensity. Although describing a non-contact brushing approach, it still might differ from actual interproximal situations. Adams et. al [1] investigated the effect of monospecies-biofilm removal using an interproximal model with various distances from the bristle tips. Analyzing the emerging bubble velocities of the different sonic toothbrushes, they estimated shear stress values between 0.5 and $0.9 \mathrm{~Pa}$, resulting in biofilm reduction up to $57 \%$ for side-to-side and $16 \%$ for oscillating-rotating toothbrushes in a distance of $0-5 \mathrm{~mm}$. Frey (2012) developed an interproximal tooth device with an integrated shear stress sensor and analyzed shear stress in interproximal distances of $0.2 \mathrm{~mm}$ using different side-to-side toothbrushes [8]. Depending on the type of toothbrush and its mode of action, shear stress values of up to $10 \mathrm{~Pa}$ were measured. However, more pronounced shear stress values with higher biofilm removal might be assessed by higher fluid flow produced by oral irrigation systems (ORS). Studies investigating the use of dental water jets indicated reduced pro-inflammatory mediators, such as IL- $1 ß$ and $\mathrm{PGE}_{2}$ and removal of salivary plaque biofilm over $99 \%$ independently of the water jet tip used [9-11]. While ORS were mainly analyzed in clinical trials, determining the outcome on reduction of bleeding, gingivitis and plaque biofilm, interproximal biofilm removal was not investigated yet $[12,13]$.

Therefore, the aim of this in-vitro study was to investigate the effect of an oral irrigator and a side-to-side toothbrush on multispecies biofilm removal using an interproximal tooth device. Moreover, treatment cycles using the ORS or sonic toothbrush were repeated in intervals of $24 \mathrm{~h}$ to simulate and analyze the effect of repeating oral hygiene patterns.

\section{Methods}

\section{Biofilm formation}

Bacterial strains were obtained from the Institute for Oral Biology, Section for Oral Microbiology and General Immunology, University of Zürich, Zürich, Switzerland. Before biofilm formation, the strains (Streptococcus mutans OMZ 918, Streptococcus oralis OMZ 607, Actinomyces naeslundii OMZ 745) were gained from precultures streaked on Columbia sheep's blood agar (CSBA) plates (bioMérieux, Marcy l'Etoile, France). Colonies were propagated planktonic in a substrate composed of $30 \%$ saliva solution and $70 \%$ modified fluid universal medium (mFUM) [14] separately on a rocker at $37^{\circ} \mathrm{C}$ in jars using gas-paks to create anaerobic conditions (GENbox anaer and GENbag anaer, bioMérieux, Marcy l'Etoile, France). Therefore, fresh saliva was gained by one healthy donor and centrifuged two times for $30 \mathrm{~min}$ by $13400 \mathrm{rpm}$. Following the opinion of the Ethics Committee of the Canton of Zurich, Switzerland, no ethical approval is needed for the donation of saliva as explained above (no. 0324/2013 and no. 50/14). The pellet was removed each time and the remaining supernatant was diluted $1: 2$ in sodium chloride $(0.9 \% \mathrm{NaCl})$ prior to sterile filtration (TPP syrenge filters with $0.2 \mu \mathrm{m}$ pores, Faust, Schaffhausen, Switzerland). The resulting saliva solution was used in all experimentations. FUM, a wellestablished tryptone-yeast based broth medium was described by Loesche et al. [15]. FUM contained (per liter of distilled water): $10 \mathrm{~g}$ of tryptone, $5 \mathrm{~g}$ of yeast extract, $3 \mathrm{~g}$ of glucose, $2 \mathrm{mg}$ of hemin, $1 \mathrm{mg}$ of menadione, $0.5 \mathrm{~g}$ of cysteine hydrochloride, $0.1 \mathrm{~g}$ of dithiothreitol, $2.9 \mathrm{~g}$ of $0.9 \% \mathrm{NaCl}, 0.5 \mathrm{~g}$ of $\mathrm{Na}_{2} \mathrm{CO}_{3}, 1 \mathrm{~g}$ of $\mathrm{KNO}_{3}, 0.45 \mathrm{~g}$ of $\mathrm{K}_{2} \mathrm{HPO}_{4}, 0.45 \mathrm{~g}$ of $\mathrm{KH}_{2} \mathrm{PO}_{4}, 0.9 \mathrm{~g}$ of $\left(\mathrm{NH}_{4}\right)_{2} \mathrm{SO}_{4}$, and $0.188 \mathrm{~g}$ of $\mathrm{MgSO}_{4} * 7 \mathrm{H} 20$. It was modified by supplementing $67 \mathrm{mmol} / \mathrm{l}$ Sørensen's buffer to a final $\mathrm{pH}$ of 7.2. Glucose was replaced by $3 \mathrm{~g}$ of a 1:1 mixture of glucose and sucrose. The modification used in this study was adopted from the Zurich biofilm protocols [14]. After approximately $6-7 \mathrm{~h}$ the bacterial solutions were adjusted to the optical density (OD550) of 1 and mixed in a tube as inoculum. To quantify the inocula per $\mathrm{ml}$, colony forming units (CFU) were plated out on CSBA plates and incubated anaerobically in jars using gas-paks $(\mathrm{t}=2 \mathrm{~d})$. In the meantime, sterile sintered hydroxyapatite discs $(\varnothing 5 \mathrm{~mm}$, Clarkson Chromatography Products, South Williamsport, USA) were incubated in $800 \mu \mathrm{l}$ of non-stimulated saliva solution for $4 \mathrm{~h}$ at gentle agitation to form a pellicle (100 rpm at room temperature). For biofilm formation, pellicle-coated discs were then placed in new 24-well polystyrene cell culture plates and incubated with $1 \mathrm{ml}$ of the prepared inocula during gentle agitation for $24 \mathrm{~h}$ in jars at $37{ }^{\circ} \mathrm{C}$ using gas-paks (GENbox anaer and GENbags anaer, bioMérieux, Marcy l'Etoile, France). Media was refreshed daily prior to treatment procedures and directly after treatment by transferring the specimens in new plates filled with fresh media (30\% saliva solution $+70 \% \mathrm{mFUM}$ ). $\mathrm{pH}$ was controlled daily in the overnight medium directly after the first media change using a $\mathrm{pH}$ meter (Mettler-Toledo Easy Five, Mettler-Toledo AG, Schwerzenbach, Switzerland).

\section{Treatment}

Specimens were divided into four groups. Three independent experiments were performed to obtain $n=18$ 
specimens per group (first experiment $n=4$, second $n=$ 6 , last experiment $n=8$ specimens per group). Each experiment consisted of three treatment days (d1, d2, d3). Prior to each treatment, measurements of the metabolic activity were performed to obtain baseline values for each specimen. Then, specimens were placed carefully into an interproximal device with 2 specimens in a distance of $0.5 \mathrm{~mm}$ face to face (Fig. 1). The brushing device for electric toothbrushes was build in a cooperation between the Institute of Fluid Dynamics, ETH Zürich and the Department of Preventive Dentistry, Periodontology and Cariology of the University of Zurich, Switzerland. For experimentation, $25 \mathrm{ml}$ water of $36{ }^{\circ} \mathrm{C}$ was pipetted into the device to cover the interproximal regions and the specimens. For the WF-group, the oral irrigator (Waterfloss, Waterpik ${ }^{\bullet}$ Sensonic WP100E) was adjusted using the JT-100E Classic Jet Tip at a $90^{\circ}$ angle towards the interproximal region as described in the manufacturer's information. The pressure control was positioned at level 10 (highest water pressure) and activated for $10 \mathrm{~s}$. Afterwards, the specimens were carefully taken from the interproximal device and restored in plates with $0.9 \% \mathrm{NaCl}$. For the WPa-group, the sonic toothbrush (Waterpik ${ }^{\odot}$ Sensonic SR-3000E) was adjusted onto the device using the respective standard brush head with a load of the brush head onto the interproximal region of $<0.9 \mathrm{~N}$ as measured for sonic toothbrushes (total load $70 \pm 5 \mathrm{~g}$ ) $[2,16]$. The brushing was performed for

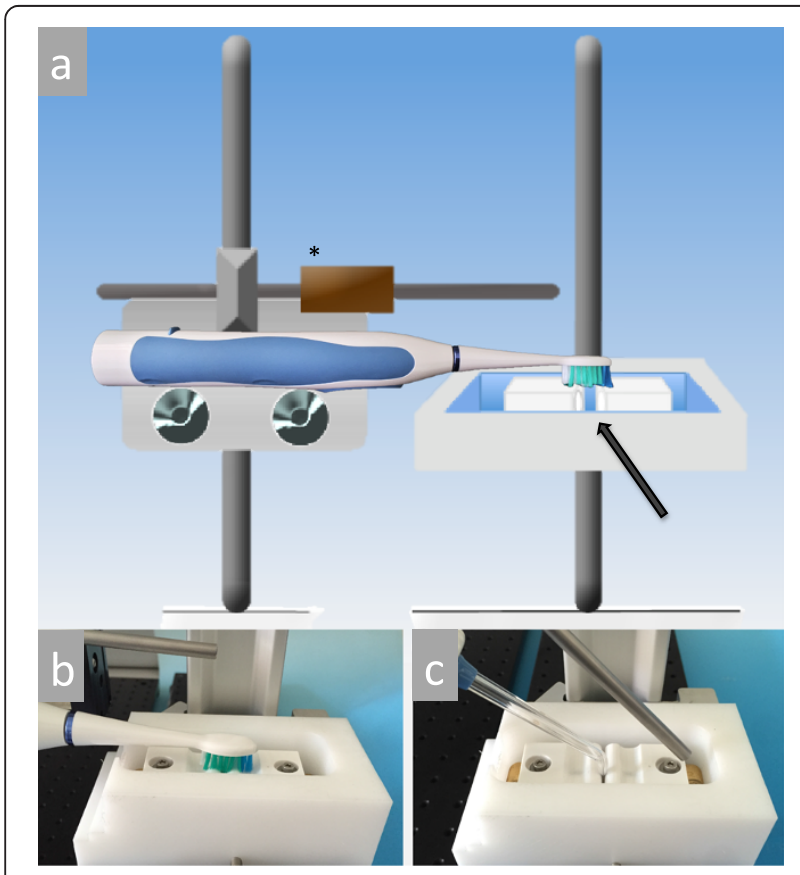

Fig. 1 a Draft of the used holding device with an adjustable load $(*)$; Interproximal specimen position within the chamber (arrow). Oral devices can be positioned perpendicularly to the fixated specimens, as illustrated in b) for the sonic toothbrush and c) for the oral irrigator
$10 \mathrm{~s}$ under static conditions. For the specimens of the WPi-group, the procedures were repeated for the inactivated brushes (power off). Specimens without treatment were used as control group (CO).

\section{alamarBlue assay and colony forming units}

Prior to experimentation, the alamarBlue assay was validated in preliminary tests [see Additional file 1]. The above-described inoculum was diluted 1:2 in phosphate buffered saline in seven series. Samples of each dilution series were determined by colony forming units, resulting in dilution series from 5.5 to $350 \times 10^{6} \mathrm{CFU} / \mathrm{ml}$. Ten samples of each series were then used for kinetic measurements. Therefore, samples were incubated in wells containing 10 vol.\% alamarBlue Cell Viability Assay Reagent (Life technologies, Zug, Switzerland) and measured in a Spectrophotometer with plate-reader at $560 \mathrm{~nm}$ excitation/585 $\mathrm{nm}$ emission at $37{ }^{\circ} \mathrm{C}$ (Spectramax M2, Molecular Devices, Bucher Biotec, Basel, Switzerland). Measurements were conducted every $15 \mathrm{~min}$ for $2 \mathrm{~h}$. The measured relative fluorescence units of each dilution were plotted against the incubation time. The findings of these preliminary tests showed constant initial rates of the enzymatic reaction within a range of approximately $30 \%$ of the total substrate conversion for each dilution curve [see Additional file 1]. To gain measurements within the range of linear increase for the current experimentations, the incubation time in the alamarBlue solution was set to $15 \mathrm{~min}$.

For experimentation, biofilm coated hydroxyapatite discs were first stored in a new well plate with $0.9 \%$ $\mathrm{NaCl}$ to avoid further growing during treatment. Then, specimens were transferred carefully into 96-well plates and incubated in $300 \mu \mathrm{l}$ alamarBlue solution containing fresh media (30\% saliva solution $+70 \%$ mFUM) with 10 vol.\% alamarBlue under anaerobic conditions. Additionally, two wells were filled with blank alamarBlue solution (without specimens) and one well was filled with centrifuged bacteria from the planktonic inocula in alamarBlue solution to gain values for the maximal metabolic activity. After $15 \mathrm{~min}, 200 \mu \mathrm{l}$ of each alamarBlue solution was pipetted into new 96 -well plates and metabolic activity was measured in a Spectrophotometer with plate-reader at $560 \mathrm{~nm}$ excitation/585 nm emission. The resulting relative fluorescence units ( $\mathrm{rfu}$ ) were defined as baseline values or pre-treatment rfu. After measurements, specimens were stored in $0.9 \% \mathrm{NaCl}$ and further experiments were conducted (treatments using the different oral devices). Then, post-treatment values were obtained by using the alamarBlue assay as described before. After rfu-measurements, specimens were transferred to fresh substrate to enable regrowth and retreated with the identical procedures $24 \mathrm{~h}$ later. Therefore, specimens were 
distributed into the same groups. Altogether, each specimen underwent three treatment cycles (d1, d2, d3).

After the last treatment and measurement using alamarBlue (d3), three specimens of each group were analyzed additionally using CFU. Therefore, specimens were vortexed in $1 \mathrm{ml} 0.9 \% \mathrm{NaCl}$ for $2 \mathrm{~min}$ and sonified for $5 \mathrm{~s}$. Each bacterial suspension was diluted in $0.9 \% \mathrm{NaCl}$ and plated out on Columbia sheep's blood agar (CSBA) plates (bioMérieux, Marcy l'Etoile, France). CSBA plates were then incubated in jars at $37{ }^{\circ} \mathrm{C}$, using gas -paks (GENbag anaer, bioMeriux, Marcy l'Etoile, France) for 2 days.

\section{Scanning electron microscopic analysis}

For SEM, two samples per group $(n=8)$ were used after the last treatment cycle (d3). Specimens were washed with $0.9 \% \mathrm{NaCl}$ solution and fixed in $4 \%$ glutaraldehyde solution (in $0.1 \mathrm{M}$ sodium potassium phosphate buffer, $\mathrm{pH}$ 7.0) for at least $24 \mathrm{~h}$. Dehydration was achieved gradually $(2 \times 15 \mathrm{~min}$ in ethanol $50 \mathrm{vol} . \%, 2 \times 15 \mathrm{~min}$ in ethanol 70 vol.\%, $2 \times 15 \mathrm{~min}$ in ethanol 80 vol.\%, $2 \times$ 15 min in ethanol $90 \%, 3 \times 20$ min in ethanol $96 \%$ and $2 \times 60 \mathrm{~min}$ in ethanol absolute). Prior to gold sputter coating the critical point drying was performed. Specimens of all groups were examined after the last periodically repeated treatment step (d3). Additionally images of specimens without bacteria were taken. Magnifications of $45 x$ were taken to image the specimen surfaces and 500x to show more detailed surface characteristics.

\section{Statistical analysis}

Metabolic activity was analyzed for each disc separately, and the ratio of post-treatment to baseline values were compared within the treatment days $(\mathrm{d} 1, \mathrm{~d} 2, \mathrm{~d} 3)$.
Analysis of the data was performed using ANOVA with the post-hoc Scheffe test, or Kruskal-Wallis with posthoc Mann-Whitney test.

\section{Results}

Median baseline rfu-values of $\mathrm{d} 1$ (all groups) resulted in $7821.8 \mathrm{rfu}$ (interquartile range $=5114.5$ ). Baseline rfuvalues of $\mathrm{d} 2$ (pre-treatment $\mathrm{rfu}$ ) showed higher metabolic activity than $\mathrm{d} 1$, irrespective of the treatment group (mean \pm SD, WF: $12045 \pm 4414$, WPa: $11832 \pm$ 4331, WPi: $10600 \pm 3362$, CO: $10508 \pm 3153$ ). Baseline rfu-values of $\mathrm{d} 3$ revealed reduced metabolic activity compared to d1 and d2 (mean \pm SD, WF: $5864 \pm 3974$, WPa: $6768 \pm 3753$, WPi: $6531 \pm 4490$, CO: $6878 \pm 4093$; Table 1). Post-treatment rfu-values were related to baseline rfu-values to calculate the residual metabolic activity in percentage. Significantly highest reduction in metabolic activity with regard to baseline was shown for the WF-group (oral irrigator) for $10 \mathrm{~s}$ for all treatment cycles (d1: $17.9 \%$, d2: $36.8 \%$ and d3: $17.2 \%$ ). The WPagroup (active sonic toothbrush) showed significantly reduced metabolic activity on $\mathrm{d} 1$, whereas no significant reduction was measured on treatment cycle $\mathrm{d} 2$ and $\mathrm{d} 3$ (d1: $58.8 \%$, d2: $85.2 \%$, d3: $79.6 \%$ ). Specimens treated with the inactive sonic toothbrush (Wpi) and untreated specimens $(\mathrm{CO})$ showed no significant reduction in biofilm activity at all (d1: WPi $82.5 \%$, CO $89.6 \%$; d2: WPi 82.5 \%, CO 90.0 \%; d3: WPi 96.3 \%, CO 116.3 \%; Fig. 2). Scanning electron microscopic images of the WF-group revealed almost biofilm-free surfaces with residual bacteria and partially shorn-off matrix on the outer areas (Fig. $3 \mathrm{a}$ and b). Images of the WPa-, WPi- and CO-group showed huge amounts of biofilm with peaks of bacterial islands and aggregates (Fig. 3). Median CFU data resulted in $1.0 \times 10^{\wedge} 6(\mathrm{WF}), 2.2 \times 10^{\wedge} 9(\mathrm{WPa}), 1.1 \times 10^{\wedge} 11(\mathrm{WPi})$

Table 1 Mean \pm SD of pre- and post-treatment in relative fluorescence units [rfu] for the different devices at time points d1-d3

\begin{tabular}{|c|c|c|c|c|c|}
\hline \multirow{2}{*}{$\frac{\text { Treatment days }}{\mathrm{d} 1}$} & \multirow{2}{*}{$\begin{array}{l}\text { Groups } \\
\text { WF }\end{array}$} & \multicolumn{2}{|c|}{ Pre-treatment [rfu] mean \pm SD } & \multicolumn{2}{|c|}{ Post-treatment [rfu] mean $\pm \mathrm{SD}$} \\
\hline & & $7077 \pm$ & 2564 & $1498 \pm$ & 1484 \\
\hline & WPa & $7384 \pm$ & 2434 & $4715 \pm$ & 2841 \\
\hline & WPi & $6832 \pm$ & 3202 & $5944 \pm$ & 3244 \\
\hline & $\mathrm{CO}$ & $6669 \pm$ & 3157 & $5930 \pm$ & 2845 \\
\hline \multirow[t]{4}{*}{$\mathrm{d} 2$} & WF & $12045 \pm$ & 4414 & $4540 \pm$ & 2451 \\
\hline & WPa & $11832 \pm$ & 4331 & $9966 \pm$ & 3414 \\
\hline & WPi & $10600 \pm$ & 3362 & $8953 \pm$ & 3788 \\
\hline & $\mathrm{CO}$ & $10508 \pm$ & 3153 & $9609 \pm$ & 3564 \\
\hline \multirow[t]{4}{*}{ d3 } & WF & $5864 \pm$ & 3974 & $885 \pm$ & 564 \\
\hline & WPa & $6768 \pm$ & 3753 & $4627 \pm$ & 2087 \\
\hline & WPi & $6531 \pm$ & 4490 & $4757 \pm$ & 1832 \\
\hline & $\mathrm{CO}$ & $6878 \pm$ & 4093 & $5979 \pm$ & 2318 \\
\hline
\end{tabular}

WF oral irrigator, WPa sonic toothbrush active, WPi sonic toothbrush inactive, CO Control 

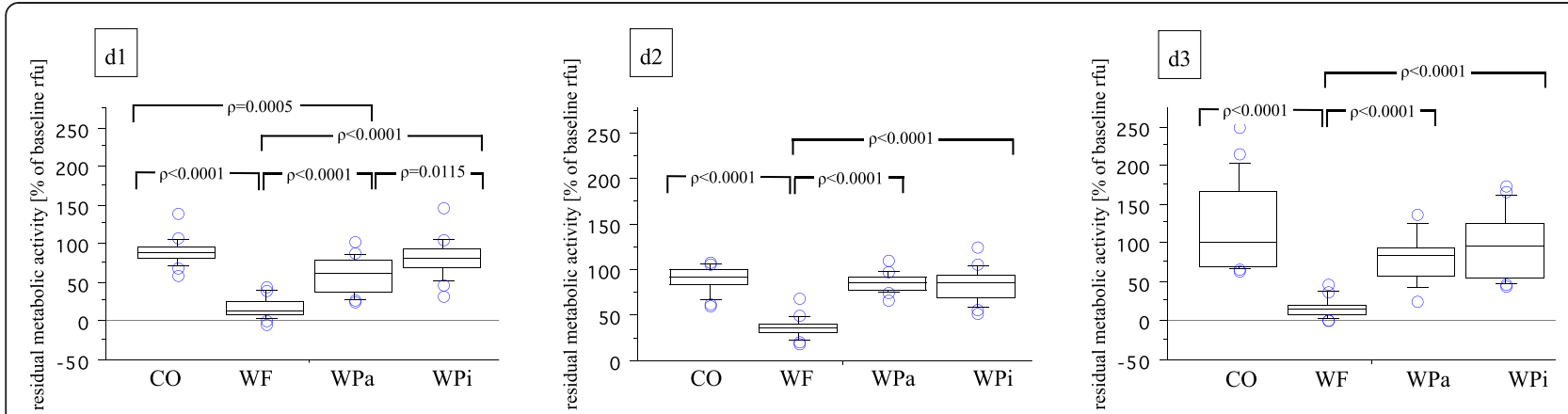

Fig. 2 Boxplots of residual metabolic activity in \% of baseline rfu-values of different experimental conditions with median (inner horizontal line), $1^{\text {st }}$ and $3^{\text {rd }}$ quartile (lower and upper box line) and $10^{\text {th }}$ and $90^{\text {th }}$ percentile (whiskers). WF $=$ oral irrigator; WPa $=$ sonic toothbrush, active; WPi $=$ sonic toothbrush, inactive; $\mathrm{CO}=$ Control. Significant differences are illustrated above corresponding boxplots

and $1.8 \times 10^{\wedge} 11(\mathrm{CO})$. Two specimens of the CO-group were uncountable $\left(>10^{\wedge} 11\right.$; Fig. 4$)$.

\section{Discussion}

Lowest residual metabolic activity of interproximal biofilms on $\mathrm{d} 1, \mathrm{~d} 2$ and $\mathrm{d} 3$ was achieved using the oral irrigator. Significant reduction in activity was also shown after treatments on $\mathrm{d} 1$ with the active sonic toothbrush. CFU data of the specimens after treatment on $\mathrm{d} 3$ mirror the same graduations between the groups. However, the analysis of intervallic treatment patterns (d1-d3) highlighted, independent of the different treatment procedures, the high re-growth rate on each specimen after $24 \mathrm{~h}$. Considering only the metabolic activity of the different groups after $24 \mathrm{~h}$ (baseline of $\mathrm{d} 2$ or $\mathrm{d} 3$, Table 1 ), there seem no differences at all. Biofilm reduction of over $63-83 \%$ using an oral irrigator results in same biofilm activity as in the control group (CO) with no treatment, after $24 \mathrm{~h}$. However, this study mainly investigated metabolic activity of biofilms. Differences in pathogenicity of treated or untreated biofilms were not analyzed. Also, results of intervallic treatment were only shown over a period of three days. Biofilm regrowth and resistance to treatment may change after longer cleaning periods.

The application time of the oral irrigator was set at $10 \mathrm{~s}$. Still, residual activity in a range of $17-37 \%$ was measured. Regarding the SEM images with the oral irrigator, shornoff matrix regions and residual biofilm areas in the outer disc regions are shown. This may be explained by the central water-jet of the oral irrigator, which seems to be very defined and does not strike the whole surface of the biofilm-coated discs to the same extent. Marginal regions of the disc, which were not in direct contact with the water-jet may result in higher amounts of residual bacteria due to less shear force. Also, the use of batch biofilms leads to bacterial adherence on all disc surfaces. The sides of the discs were not reached by oral devices and might harbor residual bacteria. The oral irrigator was applied perpendicular to the interproximal space, leading to a tangential jet to the biofilm surface. Single bacteria on the sides of the specimens may have remained untreated, however, their influence seems rather negligible due to their minor amount. Biofilm activities of $59-85 \%$ remained after non-contact cleaning with the sonic toothbrush for $10 \mathrm{~s}$. Previous studies have reported non-contact biofilm removal of more than $50 \%$ by side-to-side toothbrushes [1, 2, 17-19]. Most of these studies were analyzed microscopically after staining using a confocal laser scanning microscope. Selected areas of the treated surfaces were scanned and remaining biofilm volumes were calculated and set into relation to control groups. In contrast to the methodology used in the present study, only treated surface areas were included to the analysis. However, these studies fail to compare each specimen separately prior to and after each treatment. Microscopic analysis provides only insight to the specimens after treatment, due to irreversible staining procedures. The use of alamarBlue allows repeatable measurements. Each specimen can be analyzed at different time points and effects of intervallic treatments can be observed using the identical specimen. Its application for biofilm quantification was investigated in several studies before $[20,21]$. It was also validated for the three-species biofilm used in the experiments (see Additional file 1).

Since most investigations on the so called 'non-contact biofilm removal' were performed by directly positioned oral devices towards the center of the biofilm coated disc surfaces instead of using interproximal devices, and due to the high variety of application time, distance to specimen surfaces and biofilm models used, comparisons between single studies should be made only very carefully.

Differences between the biofilm models can also be observed with reference to the surface material used as substratum. Human enamel sections are often substituted by titanium [4], glass [1, 3, 6, 17] or hydroxyapatite discs $[2,14,18]$. The present study was performed with hydroxyapatite discs as tooth enamel analogue to avoid 


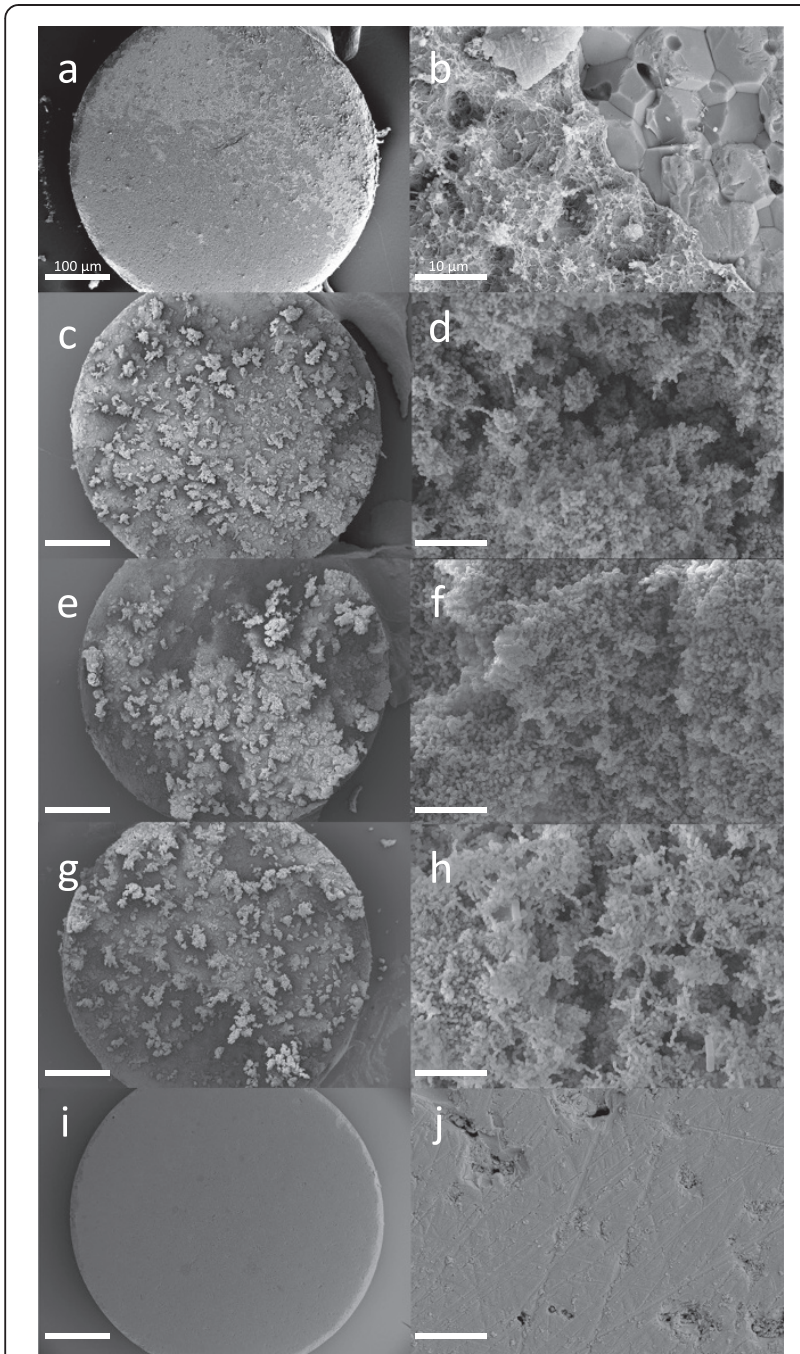

Fig. 3 SEM images of all groups after the last treatment (d3). Specimens after treatment with the oral irrigator WF $(\mathbf{a}, \mathbf{b})$, the active sonic toothbrush WPa $(\mathbf{c}, \mathbf{d})$, the inactive sonic toothbrush WPi $(\mathbf{e}, \mathbf{f})$, the control group CO without treatment $(\mathbf{g}, \mathbf{h})$ and specimens without bacteria (i,j). Areas with shorn-off biofilm are shown in $\mathbf{a}$ ) and $\mathbf{b}$ ). Magnifications of $45 \times(a, c, g, e, l$; scale bar $=100 \mu \mathrm{m})$ and 500x (b,d,f,h,j; scale bar $=10 \mu \mathrm{m}$ ) were used

possible inhomogeneous bacterial adhesion patterns along enamel cracks and fissures.

With respect to the better performance of the oral irrigator as compared to the activated sonic toothbrush one has to bear in mind that in both cases no toothpaste was involved, although by using a toothbrush this is rarely the case. The additional use of toothpaste together with the activated sonic toothbrush may have lead to further removal of biofilm due to a mechanical effect of particles from the toothpaste, and also due to further ingredients e.g. tensides. Therefore, for further studies the additional influence of toothpaste should be investigated, as well. However, it was the intention of this study to investigate

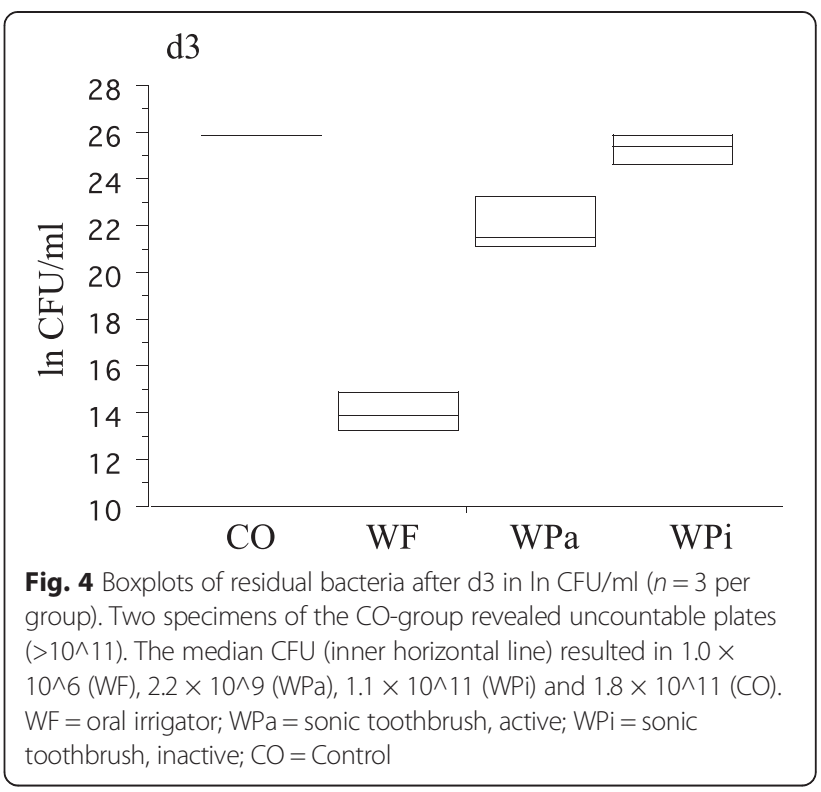

the specific influence of the devices without interference by other factors. For further research, the effect of longer intervallic cleaning procedures would help understanding the long-term effect of different oral hygiene pattern on interproximal biofilms. The comparison of noncontact brushing by interproximal devices and by noncontact brushing by directly positioned oral devices towards the biofilms would facilitate comparisons between different study models.

\section{Conclusions}

Based on the results, cleaning of interproximal regions by hydrodynamic flow of an oral irrigator may achieve more effective removal of interproximal biofilm compared to sonic toothbrushes.

\section{Additional file}

Additional file 1: Validation of the alamarBlue assay. (DOCX 194 kb)

\section{Abbreviations}

CFU: Colony forming units; CO: Control specimens (biofilm-coated specimens without treatment); d1: First day of treatment; $d 2$ : Second day of treatment; d3: Third day of treatment; mFUM: modified fluid universal medium;

$\mathrm{NaCl}$ : Sodium chloride; ORS: Oral irrigation systems; rfu: Relative fluorescence units; SEM: Scanning electron microscope; WF: Waterpik ${ }^{\oplus}$ Sensonic WP-100E: oral irrigator; WPa: Waterpik ${ }^{\circledR}$ Sensonic SR-3000E: sonic toothbrush, active; WPi: Waterpik ${ }^{\circledast}$ Sensonic SR-3000E: sonic toothbrush, inactive.

\section{Competing interests}

The authors declare that they have no competing interests.

\section{Authors' contributions}

Dr. Tawakoli conceived this research and was the main investigator in this study. Mrs. Sauer and Mr. Becker helped with the performance of the study 
and with methodological issues. Dr. Buchalla and Dr. Attin supervised the study and critically revised the manuscript. All authors read and approved the final manuscript.

\section{Acknowledgement}

The authors would like to thank Beatrice Sener of the Clinic for Preventive Dentistry, Periodontology and Cariology for her great support with the SEM images.

\section{Author details}

${ }^{1}$ Clinic of Preventive Dentistry, Periodontology and Cariology, University of Zurich, Center of Dental Medicine, 8032 Zurich, Switzerland. ${ }^{2}$ Department for Conservative Dentistry and Periodontology, University Medical Center, University of Regensburg, Regensburg, Germany.

Received: 9 April 2015 Accepted: 29 July 2015

Published online: 05 August 2015

\section{References}

1. Adams H, Winston MT, Heersink J, Buckingham-Meyer KA, Costerton JW, Stoodley P. Development of a laboratory model to assess the removal of biofilm from interproximal spaces by powered tooth brushing. Am J Dent. 2002;15 Spec No:12B-7B

2. Hope CK, Wilson M. Comparison of the interproximal plaque removal efficacy of two powered toothbrushes using in vitro oral biofilms. Am J Dent. 2002;15 Spec No:7B-11B.

3. Verkaik MJ, Busscher HJ, Rustema-Abbing M, Slomp AM, Abbas F, van der Mei HC. Oral biofilm models for mechanical plaque removal. Clin Oral Investig. 2010;14:403-9.

4. Schmidt JC, Astasov-Frauenhoffer M, Hauser-Gerspach I, Schmidt JP, Waltimo T, Weiger R, et al. Efficacy of various side-to-side toothbrushes for noncontact biofilm removal. Clin Oral Investig. 2014;18:793-800.

5. Brambilla E, Cagetti MG, Belluomo G, Fadini L, Garcia-Godoy F. Effects of sonic energy on monospecific biofilms of cariogenic microorganisms. Am Dent. 2006;19:3-6.

6. Heersink J, Costerton WJ, Stoodley P. Influence of the Sonicare toothbrush on the structure and thickness of laboratory grown Streptococcus mutans biofilms. Am J Dent. 2003;16:79-83.

7. Stanford CM, Srikantha R, Kirchner HL, Wu CD. Removal of supragingival plaque in an intraoral model by use of the Sonicare toothbrush. J Int Acad Periodontol. 2000;2:115-9.

8. Frey S. Characterization of the inter-proximal flow generated by sonic toothbrushes measured experimentally by a shear-stress sensor. Institute of Fluid Dynamics ETH Zürich. 2012.

9. Al-Mubarak S, Ciancio S, Aljada A, Mohanty P, Ross C, Dandona P. Comparative evaluation of adjunctive oral irrigation in diabetics. J Clin Periodontol. 2002;29:295-300.

10. Cutler CW, Stanford TW, Abraham C, Cederberg RA, Boardman TJ, Ross C. Clinical benefits of oral irrigation for periodontitis are related to reduction of pro-inflammatory cytokine levels and plaque. J Clin Periodontol. 2000;27:134-43.

11. Gorur A, Lyle DM, Schaudinn C, Costerton JW. Biofilm removal with a dental water jet. Compend Contin Educ Dent. 2009;30 Spec No 1:1-6.

12. Barnes CM, Russell CM, Reinhardt RA, Payne JB, Lyle DM. Comparison of irrigation to floss as an adjunct to tooth brushing: effect on bleeding, gingivitis, and supragingival plaque. J Clin Dent. 2005;16:71-7.

13. Cobb CM, Rodgers RL, Killoy WJ. Ultrastructural examination of human periodontal pockets following the use of an oral irrigation device in vivo. J Periodontol. 1988;59:155-63.

14. Thurnheer T, Gmur R, Guggenheim B. Multiplex FISH analysis of a six-species bacterial biofilm. J Microbiol Methods. 2004:56:37-47.

15. Loesche WJ, Hockett RN, Syed SA. The predominant cultivable flora of tooth surface plaque removed from institutionalized subjects. Arch Oral Biol. 1972;17:1311-25

16. Wiegand A, Burkhard JP, Eggmann F, Attin T. Brushing force of manual and sonic toothbrushes affects dental hard tissue abrasion. Clin Oral Investig. 2013;17:815-22.

17. Busscher HJ, Jager D, Finger G, Schaefer N, van der Mei HC. Energy transfer, volumetric expansion, and removal of oral biofilms by non-contact brushing. Eur J Oral Sci. 2010;118:177-82.
18. Hope CK, Petrie A, Wilson M. In vitro assessment of the plaque-removing ability of hydrodynamic shear forces produced beyond the bristles by 2 electric toothbrushes. J Periodontol. 2003;74:1017-22.

19. Schmidt JC, Zaugg C, Weiger R, Walter C. Brushing without brushing?-a review of the efficacy of powered toothbrushes in noncontact biofilm removal. Clin Oral Investig. 2013;17:687-709.

20. Jiang LM, Hoogenkamp MA, van der Sluis LW, Wesselink PR, Crielaard W, Deng DM. Resazurin metabolism assay for root canal disinfectant evaluation on dual-species biofilms. J Endod. 2011;37:31-5.

21. Peeters E, Nelis HJ, Coenye T. Comparison of multiple methods for quantification of microbial biofilms grown in microtiter plates. J Microbiol Methods. 2008;72:157-65.

\section{Submit your next manuscript to BioMed Central and take full advantage of:}

- Convenient online submission

- Thorough peer review

- No space constraints or color figure charges

- Immediate publication on acceptance

- Inclusion in PubMed, CAS, Scopus and Google Scholar

- Research which is freely available for redistribution 